\title{
IDENTIFICAÇÃO DE TENDÊNCIAS EM NÍVEIS FREÁTICOS FRENTE ÀS ANOMALIAS CLIMÁTICAS OCORRIDAS ENTRE 2014 E 2016 EM ÁREA DE CONSERVAÇÃO DE CERRADO NA REGIÃO HIDROGRÁFICA DO MÉDIO PARANAPANEMA/SP
}

\author{
IDENTIFICATION OF TRENDS AT PHREATIC LEVELS FACING \\ CLIMATE ANOMALIES OCCURRED BETWEEN 2014 AND 2016 \\ IN CERRADO CONSERVATION AREA IN THE HYDROGRAPHIC \\ REGION OF MIDDLE PARANAPANEMA / SP - BRAZIL
}

\author{
IDENTIFICACIÓN DE TENDENCIAS EN NIVELES FREÁTICOS FRENTE \\ A LAS ANOMALÍAS CLIMÁTICAS OCURRIDAS ENTRE 2014 Y 2016 \\ EN UNA ÁREA DE CONSERVACIÓN DE CERRADO EN LA REGIÓN \\ HIDROGRÁFICA DEL MEDIO PARANAPANEMA/ SP - BRASIL
}
Rodrigo Lilla Manzione - Universidade Estadual Paulista Júlio de Mesquita Filho - Faculdade de Ciências e Engenharia de Tupã - São Paulo- Brasil
rlmanzione@gmail.com

\begin{abstract}
Resumo
Aliar as dimensões temporais e espaciais na análise de fenômenos pode trazer respostas que a análise espacial de dados geográficos sozinha não revelaria. Entretanto, reunir escalas que não variam da mesma maneira, como 0 espaço e o tempo, não é rotina em geociências devido à complexa estrutura desses dados. 0 objetivo desse trabalho foi investigar o comportamento dos recursos hídricos subterrâneos frente às anomalias climáticas ocorridas no estado de São Paulo entre 2013-2016 em área representativa da região hidrográfica do Médio Paranapanema (UGRHI-17), no tempo e no espaço. Para isso, implantou-se na Estação Ecológica de Santa Bárbara (EEcSB), Águas de Santa Bárbara/SP, uma rede de monitoramento hidrometeorológica para coletar dados climatológicos e de níveis freáticos em 32 poços entre setembro de 2014 e agosto de 2016. A análise de séries temporais revelou que os estímulos climáticos influenciaram o comportamento dos níveis freáticos, indicando tendências de elevação no período estudado. Já a análise espacial revelou padrões diferenciados de elevação entre bacias da EEcSB, principalmente em função do uso da terra. Essas informações são importantes para a gestão dos recursos hídricos subterrâneos, planejamento de atividades que dependam da água e estudos sobre a capacidade de suporte de áreas quanto aos seus recursos naturais. Palavras-chave: Séries temporais, águas subterrâneas, monitoramento, PIRFICT.
\end{abstract}

\begin{abstract}
Allying temporal and spatial dimensions at phenomena analysis can bring answers that traditional geographical spatial data analysis alone would not reveal. However, gathering scales that do not vary in the same way, such as space and time, is not routine in geosciences due to the complex structure of these data.The aim of this study was to investigate the behavior of groundwater resources against the climatic anomalies occurred in the State of São Paulo between 2013-2016 in a representative area of the Médio Paranapanema hydrographic region (UGRHI-17), in time and space. A monitoring network of water table depths and an automatic climatological station were installed in the Ecological Station of Santa Bárbara, municipality of Águas de Santa Bárbara (SP), Brazil, to collect groundwater and climatological data between September 2014 and August 2016 at 32 wells. Time series modelling showed that the climatic inputs influenced the behavior of the groundwater levels,
\end{abstract}

ISSN: 1984-8501 Bol. Goia. Geogr. (Online). Goiânia, v. 38, n. 1, p. 68-85, jan./abr. 2018 
indicating elevation trends in the studied period. On the other hand, the spatial analysis revealed differentiated patterns of elevation between EEcSB basins, mainly as a land use function.This information is important for groundwater management, water dependable activities planning and studies about the natural resources capacity of specific areas.

Keywords: Time series, groundwater, monitoring, PIRFICT.

\section{Resumen}

Aliar las dimensiones temporales y espaciales en el análisis de fenómenos puede traer respuestas que el análisis espacial de datos geográficos por sí solas no revelaría. Sin embargo, reunir escalas que no varían de la misma manera, como el espacio y el tiempo, no es rutina en geociencias debido a la compleja estructura de esos datos.El objetivo de este trabajo fue investigar el comportamiento de los recursos hídricos subterráneos frente a las anomalías climáticas ocurridas en el Estado de São Paulo entre 2013-2016 en un área representativa de la región Hidrográfica del Medio Paranapanema (UGRHI-17), en el tiempo y en el espacio. Se llevó a cabo en la estación ecológica de Santa Bárbara (EEcSB), en el municipio de Águas de Santa Bárbara (SP/Brasil), una red de monitoreo del agua subterránea y una estación meteorológica automática para recoger datos sobre el clima y los niveles de agua subterránea entre septiembre de 2014 y agosto de 2016. El análisis de series temporales mostró que los estímulos climáticos influyeron en el comportamiento de los niveles de agua subterránea, lo que indica las tendencias de elevación durante el período de estudio. Por otra parte, el análisis espacial reveló estándares diferenciados de elevación entre cuencas de la $\mathrm{EEcSB}$, principalmente en función del uso de la tierra. Esta información es importante para la gestión de los recursos de aguas subterráneas, la planificación de actividades que dependen del agua y y los estudios sobre la capacidad de soporte de áreas con respecto a sus recursos naturales.

Palabras clave: Series temporales, agua subterránea, monitoreo, PIRFICT.

\section{Introdução}

Uma linha comum em estudos ambientais é a necessidade metodológica de ferramentas capazes de descrever e prever estes processos complexos e, tipicamente, de alta dimensionalidade. Cressie e Holan (2011) descrevem novas abordagens para a modelagem neste contexto, destacando que entre elas o estudo de séries temporais ambientais é fundamental para o objetivo maior de sustentabilidade e adaptação. A gestão dos recursos hídricos requer o uso de técnicas de modelagem que reconheçam a variabilidade, tanto temporal quanto espacial, para que as análises, diagnósticos e recomendações realizadas não percam informações valiosas na resolução de problemas complexos. Saber como e, em última análise, porque os processos ambientais mudam ao longo do tempo dá aos governos e protetores dos comuns um meio racional para tomada de decisão.

Para Cressie e Wikle (2011), a causalidade é o "santo graal" da ciência, buscando inferir sobre relações de causa e efeito, ou o "porquê" das coisas. Esse pensamento é seguido pelo "quando", já que uma causa sempre precede seu efeito. Continuando nessa linha, definir "onde" as coisas acontecem denota o caráter histórico e geográfico do evento. Então, 
para que uma boa resposta do "porquê" seja encontrada, ela deve ser acompanhada do "quando" e do "onde". Incorporar a dimensão temporal e espaciais dentro de um mesmo modelo de variabilidade continua sendo um desafio para os geocientistas, uma vez que as variações não ocorrem na mesma escala. Enquanto no espaço se lida com metros, quilômetros (métricas de distância), no tempo se lida com dias, meses, anos, décadas (intervalos temporais). A análise de séries temporais associada à análise espacial de dados geográficos possibilita acessar as dimensões temporais espaciais da variabilidade do fenômeno em estudo, e dados de monitoramento quando coletados em redes geoespaciais permitem que essas técnicas sejam exploradas.

O monitoramento das águas subterrâneas é instrumento fundamental para a avaliação das condições que este meio natural se encontra, e, posteriormente, poder tomar medidas preventivas e/ou proativas para o predomínio da qualidade e quantidade, buscando desenvolver o uso sustentável junto a uma ação integrada de gerenciamento (Mestrinho, 2008). Um bom programa de monitoramento irá incluir o planejamento, execução, interpretação, avaliação dos resultados e reavaliação da eficiência do programa. Os pontos de monitoramento devem ser locados em locais com características hidrogeológicas conhecidas, e devem ser pontos fixos para que possa ter uma série histórica de dados para melhor compreensão da dinâmica que ali opera. A coleção de dados de monitoramento requer tratamento com métodos estatísticos, cartográficos, gráficos e modelagem. Portanto após um período de monitoramento os dados são analisados estatisticamente e as incertezas são consideradas, e com isso pode-se analisar do ponto de vista técnico-ambiental e socioeconômico para a tomada das devidas decisões e revisão dos objetivos da rede (Moore, 2012).

Em períodos de escassez hídrica tem-se a diminuição da oferta, em paralelo o aumento da demanda, seja pelo setor doméstico, industrial ou agrícola. Sabendo da importância que a água exerce para a sociedade, tanto para abastecimento doméstico, atividades industriais e agricultura, os recursos hídricos subterrâneos despertam grande interesse ambiental em relação a sua conservação. Assim, o volume explorável de um aquífero é uma variável de decisão a ser determinada como parte de um plano de gestão do sistema de águas de uma região. Só que para isso é necessário conhecer a dinâmica das águas subterrâneas e identificar os processos que influenciam a oscilação dos seus níveis. O monitoramento da água 
disponível em um aquífero possibilita diagnosticar o atual estado do aquífero, em relação a estados passados, para tomar as devidas medidas em relação a modificações causadas por efeitos naturais e/ou antrópicos.

Na região Hidrográfica do Médio Paranapanema (UGRHI-17), as águas subterrâneas são fonte de abastecimento de diversas cidades, de inúmeras nascentes e dos principais rios que suportam sistemas agrícolas e florestais, além de remanescentes de Cerrado (CBH-MP, 2010). Assim, o objetivo desse trabalho foi verificar os efeitos do clima e da sazonalidade no processo de oscilação dos níveis freáticos a partir de dados de monitoramento agrohidrometeorológico coletados em uma rede geoespacial entre 2014 e 2016 em área representativa da UGRHI-17. Para isso, procurou-se incorporar as dimensões temporais e espaciais do processo de oscilação das águas subterrâneas para inferir sobre a dinâmica do Sistema Aquífero Bauru (SAB), um dos principais mananciais subterrâneos disponíveis na UGRHI-17.

2 Área de estudo

\subsection{Estação ecológica de Santa Bárbara}

A Estação Ecológica de Santa Bárbara (EEcSB) está localizada à beira da Rodovia SP 261 - km 58, sob as coordenadas 2248’59" Sul e 49¹4'12" Oeste, no município de Águas de Santa Bárbara/SP (Figura 1). Foi regulamentada pelo Decreto 22.337 de 07 de junho de 1984 que instituiu sua formação com uma área de 4.371 hectares dentro dos limites da Floresta Estadual de Santa Bárbara, dos quais apresentam uma área 2.712 hectares de vegetação nativa (Cerrado, brejos e mata de galeria) dividindo o espaço com campos antrópicos, vegetação em estágio de regeneração, mata ciliar e áreas de reflorestamento com Pinus e Eucalipto. 


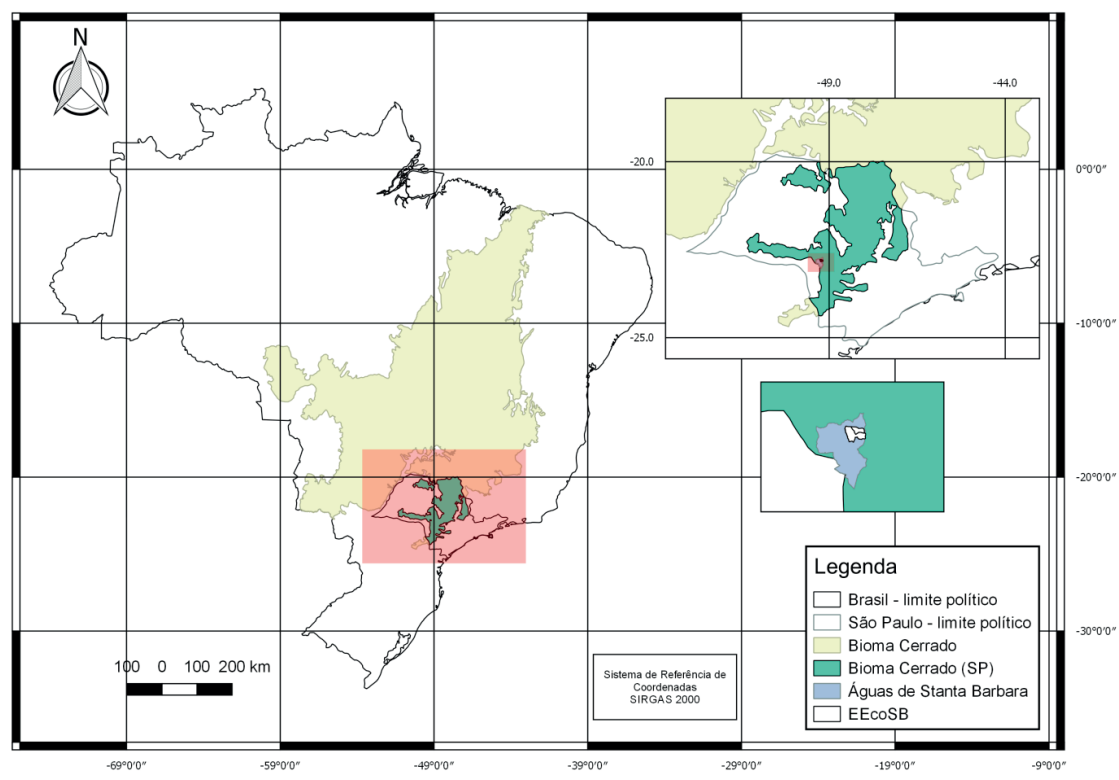

Figura 1 - Localização da EEcSB e da Floresta Estadual nos limites do município de Águas de Santa Bárbara (SP)

Fonte: Elaborado por Rodrigo Lilla Manzione.

As formações geológicas na região perpassamos arenitos da Formação Adamantina e da Formação Marília, pertencentes ao Grupo Bauru, com predomínio da Formação Adamantina nos domínios da EEcSB (CPRM, 2006; Melo; Durigan, 2011). A EEcSB encontra-se localizada na Bacia Sedimentar do Paraná (morfoestrutura) e no Planalto Ocidental Paulista (morfoescultura) conforme descrito em Ross e Moroz (1996), possuindo como formas de relevo predominantemente colinas amplas e baixas, com altimetria em torno de 600 e $680 \mathrm{~m}$. Os principais tipos de solo presentes na EEcSB são Latossolos Vermelhos (LV56) e Argissolos Vermelhos-Amarelo e Vermelho (PVA10) eutróficos e distroférricos com textura arenosa/média e Nitossolos (NV1) eutroférricos com textura argilosa (Oliveira et al., 1999).

Segundo a classificação de Koeppen, o clima característico da região é tropical subúmido (Cwa - clima quente com inverno seco). A temperatura média anual está em torno de $18^{\circ} \mathrm{C}$, apresentando temperaturas de $16^{\circ} \mathrm{C}$ no 
mês mais frio e $23^{\circ} \mathrm{C}$ no mês mais quente (Cepagri, 2016). As precipitações anuais estão em torno de 1000 e $2086 \mathrm{~mm}$, podendo chegar a $30 \mathrm{~mm}$ mensais no inverno (Melo; Durigan, 2011).

\subsection{Características climáticas do período estudado}

O verão de 20013/2014 foi um dos mais secos já registrados no estado de São Paulo (Coelho; Cardoso; Firpo, 2016). Isso trouxe efeitos diretos no ciclo hidrológico, diminuindo a recarga dos aquíferos e a produção de água de nascentes, contribuindo ainda mais para o quadro de escassez hídrica. Em contrapartida, em 2015 e 2016, o mundo presenciou um dos fenômenos El Niño Oscilação Sul (ENOS) mais fortes já registrados. Os impactos dos eventos El Niño e La Niña acarretam em dificuldades evidentes sobre o Sul da América do Sul nos setores hidrológico (Boulanger et al., 2005) e agrícola (Podestá et al., 1999), particularmente sobre o nordeste da Argentina, a parte mais meridional do Brasil e do Uruguai (Penalba; Rivera, 2016). O excesso de precipitação associado aos eventos El Niño contribui para o excesso de condições observadas no teor de água do solo (Spescha et al., 2004) e um aumento de cerca de 10\% no armazenamento de água no solo (Penalba et al., 2014). Nesse último evento, diversos locais tiveram seus regimes de precipitação alterados, como no interior do estado de São Paulo onde áreas de clima subtropical tipicamente com verão chuvoso e inverno seco tiveram um inverno chuvoso, favorecendo a recuperação dos mananciais superficiais e subterrâneos já em 2016.

\subsection{Dados disponíveis}

Partindo do pressuposto que após o verão seco de 2013-2014 os níveis das águas subterrâneas estariam suficientemente mais baixos do que o normal no final do inverno de 2014, imaginou-se que a partir da implantação de uma rede de monitoramento de níveis freáticos em área de estudo piloto seria capaz de caracterizar o comportamento das águas subterrâneas após essa anomalia climática. Foram perfurados manualmente 32 poços próximos às principais drenagens da EEcSB (Figura 2). Esses poços foram visitados com uma frequência mensal, entre julho de 2014 e setembro de 2016, e os níveis freáticos registrados com uso de um medidor de nível tipo carretel, criando um banco de dados de 26 meses sobre o comportamento do aquífero na EEcSB. 
Os dados climatológicos utilizados nesse estudo foram séries de precipitação e evapotranspiração potencial, oriundas de uma estação climatológica compacta automática (ECA) instalada na área de estudo em julho de 2014. Essa ECA foi reprogramada para coletar dados diários, em frequência horária, de velocidade e direção do vento, radiação solar, temperatura, umidade relativa e precipitação. Além disso, a evapotranspiração potencial é calculada pelo método padronizado da ASCE (American Society of Civil Engineers) (Allen et al., 1998). Os dados utilizados para modelagem das séries temporais perfazem dois anos hidrológicos contados a partir da primavera, de setembro de 2014 a agosto de 2016. No primeiro ano hidrológico (2014/15) a precipitação foi $1280,20 \mathrm{~mm}$ e no segundo (2015/16) 1756,80 mm.

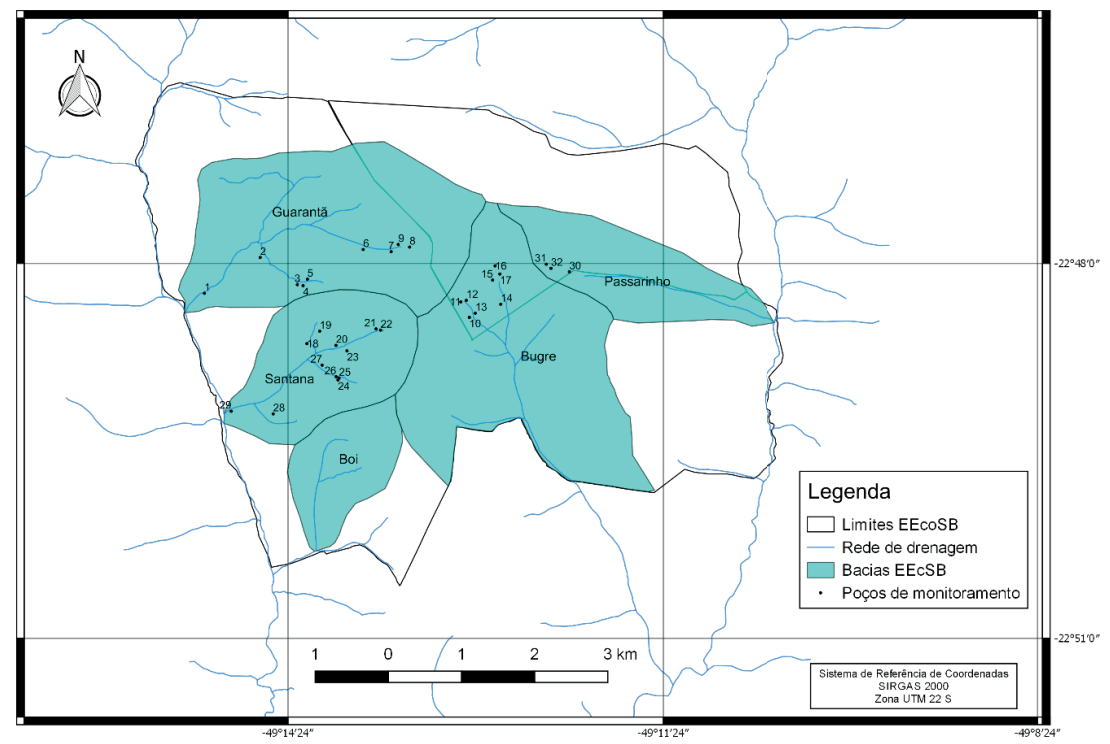

Figura 2 - Localização dos poços de monitoramento nas principais bacias hidrográficas da EEcSB e da Floresta Estadual. 


\section{Modelagem dos dados}

\subsection{Modelagem dos dados de séries temporais de monitoramento}

O comportamento de um sistema linear de entrada e saída pode ser completamente caracterizado por sua função de impulso e resposta (IR) (Ziemer; Tranter; Fannin, 1998; Von Asmuth; Bierkens; Maas, 2002). Para altura de lençol freático, a relação dinâmica entre a precipitação incidente em uma área e a resposta nos níveis freáticos pode ser explicada por modelos de função de transferência de ruído (FTR) (Knotters, 2001). Em modelos de FTR, um ou mais componentes determinísticos de transferência e um ruído são determinados como componentes aditivos. Os componentes de transferência descrevem a parte do processo de oscilação dos níveis que pode ser explicada pelas séries de entrada (precipitação, evapotranspiração, bombeamento, abstrações, fluxo do rio, entre outros) a partir de uma transformação linear dessas séries de entrada. O modelo de ruído descreve a estrutura autorregressiva das diferenças entre os níveis observados e a soma dos componentes de transferência. A entrada do modelo de ruído é uma série de perturbações independentes e identicamente distribuídas, com média zero e variância finita e constante, que é o ruído branco. Nesse estudo utilizou-se o modelo PIRFICT (Predefined Impulse Response Function In Continuous Time ou Função de Impulso e Resposta Pré-definida Em Tempo Contínuo), que é uma alternativa a modelos FTR em intervalos de tempo discretos apresentada por Von Asmuth, Bierkens e Maas (2002). No modelo PIRFICT o pulso em bloco de entrada é transformado em uma série de saída por uma função de transferência em tempo contínuo, sendo que os coeficientes dessa função não dependem da frequência de observação. Assumindo-se linearidade no sistema, uma série de alturas de lençol freático é uma transformação de uma série de precipitação excedente. Essa transformação é completamente governada pela função IR. Para o caso de um sistema linear simples, sem perturbações freáticas, que é influenciado somente pela precipitação excedente, o modelo FTR pode ser usado para descrever a relação entre alturas de lençol freático e a precipitação excedente (Von Asmuth; Bierken; Maas, 2002).

Modelos FTR são identificados através da escolha de funções matemáticas que descrevam a relação de IR e a estrutura autorregressiva 
do ruído. Essa identificação pode ser feita interativamente (usando estruturas de correlação contidas nos dados disponíveis e diagnósticos sobre modelos) ou fisicamente (baseado em conhecimentos prévios sobre o comportamento do sistema sob análise). Seguindo a identificação física do sistema, a função IR descreve a maneira com que cada lençol freático responderá a um impulso causado pela precipitação. A esse respeito, pode-se fazer uma analogia a um hidrograma unitário (Von Asmuth; Maas, 2001), no qual após um evento de precipitação haverá mudanças no fluxo de base e aumento no escoamento superficial, subsuperficial e subterrâneo. Foi adicionado aos parâmetros do modelo um parâmetro de tendência linear (PTL) para verificar o comportamento dos níveis freáticos ao longo do período monitorado. As análises do modelo PIRFICT são realizadas utilizando o software Menyanthes (Von Asmuth et al., 2012).

\subsection{Análise espacial de dados geográficos}

Segundo Stein, Staritisky e Bouma (1991), existem duas abordagens metodológicas principais para integração da dimensão temporal com a dimensão espacial. O primeiro método chamado "calcular primeiro/ interpolar depois (CP/ID)" consiste em calcular primeiro o modelo de dados pontualmente e interpolar depois seus resultados espacialmente, enquanto o segundo método chamado "interpolar primeiro/calcular depois (IP/CD)" seria basicamente interpolar primeiro os dados de entrada do modelo e calcular depois o modelo de dados de forma espacial. Em ambos contextos a análise geoestatística permite explorar a continuidade espacial de processos naturais e interpolar os dados com mínimos desvios e máxima precisão (Stein; Staritisky; Bouma, 1991; Becchini et al., 2000). Manzione (2007) propôs uma extensão à análise de séries temporais utilizando o modelo PIRFICT para a dimensão espacial através da interpolação geoestatística de parâmetros do modelo ou mesmo características do nível freático estimados a partir de simulações.

Nesse estudo, seguindo a abordagem CP/ID, realizou-se essa análise para tendências de elevação (parâmetro PTL do modelo PIRFICT) dos níveis freáticos durante o período estudado, que, posteriormente, foram interpolados geoestatisticamente. A análise geoestatística seguiu a metodologia descrita em Journel e Huijbregts (1978) e Yamamoto e Landim (2013): variografia, interpolação dos dados por krigagem ordinária e validação cruzada dos resultados. 


\section{Resultados e discussão}

De forma geral, Shapoori et al. (2015) destacam que os modelos de FTR simulam uma saída observada no modelo em determinado instante no tempo como uma ponderação de dados de forças recentes que estejam influenciando os níveis (p. ex. a função de transferência), mais um termo de correlação para a saída simulada não explicada pelas forças reinantes (p. ex. o ruído). Ajustado o modelo PIRFICT para os 32 poços de monitoramento da EECSB com um parâmetro de tendência linear para verificar a taxa de elevação dos níveis no período, pode-se verificar uma boa calibração e tendências de elevação dos níveis no período. A Tabela 1 apresenta os resultados das calibrações e dos parâmetros calculados.

Tabela 1 - Estatísticas médias das calibrações do modelo PIRFICT nos

32 poços de monitoramento entre 05/09/2014 e 02/09/2016

\begin{tabular}{ccccccccccc}
\hline & EVP & RMSE & RMSI & NDL & $\boldsymbol{A}$ & $\boldsymbol{a}$ & $\boldsymbol{n}$ & $\mathrm{e}$ & $\boldsymbol{P T L}$ & ruído \\
\hline Média & 93,38 & 0,10 & 0,09 & $-3,16$ & 300,50 & 0,01 & 1,13 & 0,20 & 1,11 & 15.85 \\
\hline
\end{tabular}

$E V P=$ variância explicada pelo modelo $(\%) ; R M S E=$ raiz do erro médio quadrático $(\mathrm{m}) ; R M S I=$ raiz da inovação média quadrática $(\mathrm{m}) ; N D L=$ nível da drenagem local $(\mathrm{m}) ; A=$ resistência a drenagem (dias); $a=$ porosidade (1/dias); $n=$ número de reservatórios lineares (-); $e=$ fator de evapotranspiração (-); PTL = parâmetro de tendência linear (m).

Fonte: Organizado pelo autor

A variância explicada pelo modelo $(E V P)$ é uma medida semelhante ao $R^{2}$, refletindo a porcentagem de quanto o modelo explica essa variação a partir das séries de entrada definidas. Os ajustes foram considerados excelentes, acima de $90 \%$ em 28 poços. As exceções foram alguns poços na Bacia do Bugre, mas mesmo assim tiveram bons ajustes, superiores a 75\%. Os valores da raiz do erro médio quadrático (RMSE) foram considerados baixos, variando de 7 a $22 \mathrm{~cm}$. O mesmo se aplica aos valores da raiz da inovação média quadrática (RMSI), que variaram de 4 a $14 \mathrm{~cm}$. Segundo Von Asmuth e Bierkens (2005) as inovações são uma maneira mais robusta de examinar os ajustes, já que calculam os erros médios entre um instante no tempo e o instante anterior. Essas três medidas referem-se aos ajustes estatísticos do modelo a cada série de dados. A Figura 3 mostra um exemplo de ajuste para cada uma das bacias estudadas. Todas as bacias apresentam algum poço com níveis superficiais (menores que 1 metro da superfície). Os níveis mais profundos monitorados foram na Bacia do Santana. 

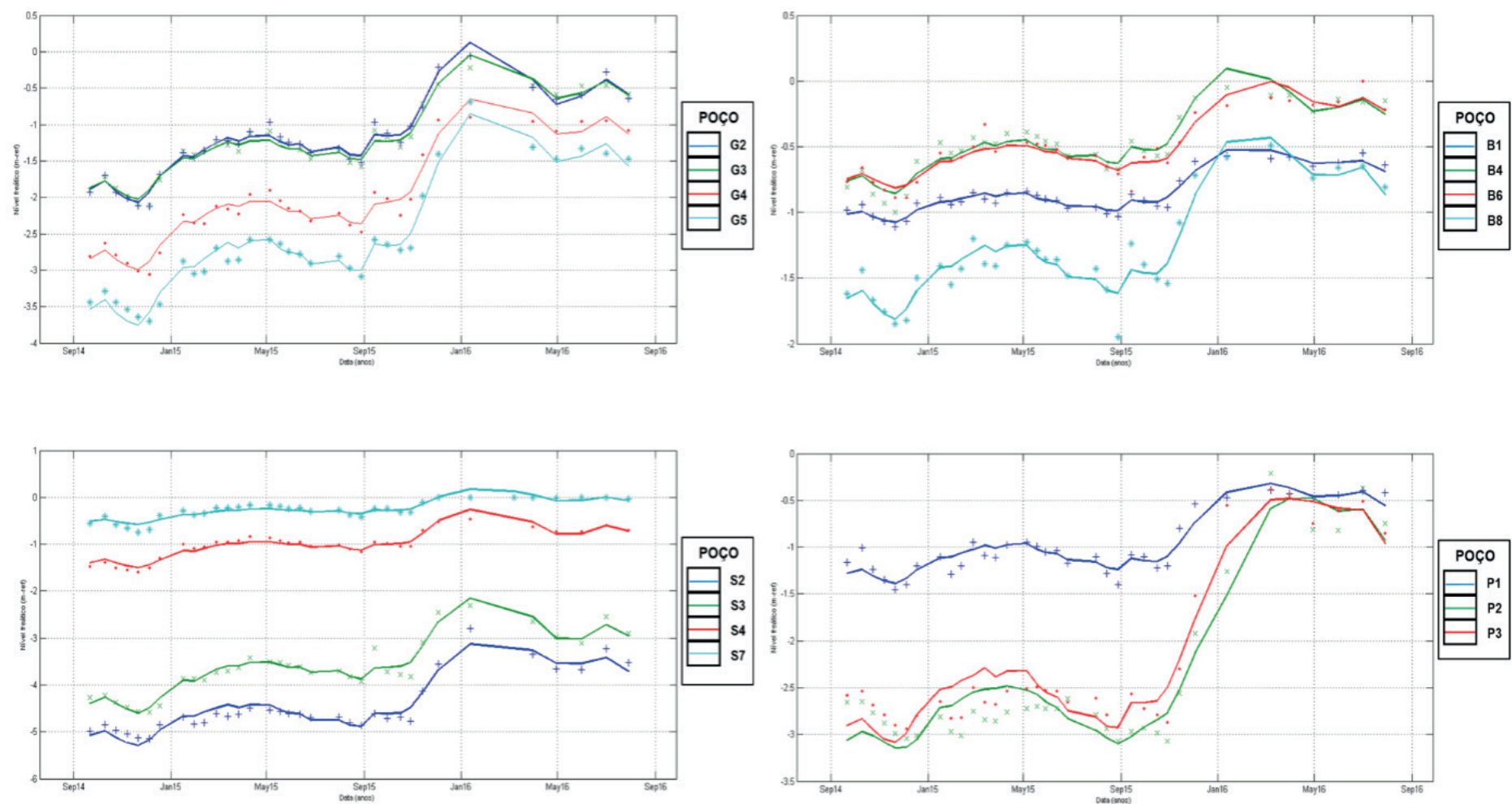

Figura 3 - Exemplos de ajustes do modelo PIRFICT para os poços monitorados entre setembro de 2014 e agosto de 2016nas bacias do Guarantã (G), Bugre (B), Santana (S) e Passarinho (P) 
Uma característica comum em todos os poços foi a elevação nos níveis mais pronunciados a partir de setembro de 2015. A presença do fenômeno ENOS que influenciou fortemente o regime de chuvas no Brasil e no Sudeste do país, alterou sensivelmente o comportamento do lençol freático, chegando a quase saturação da zona vadosa em alguns momentos. Apesar disso, o modelo PIRFICT foi capaz de recriar a série de entrada dos dados a partir das variáveis exógenas precipitação e evapotranspiração. $\mathrm{O}$ $P T L$ calculado para cada poço foi positivo em todos os casos, variando de alguns centímetros $(44 \mathrm{~cm})$ a alguns metros $(2,30 \mathrm{~m})$. Não foi verificado um padrão de elevação entre as áreas de Cerrado e as áreas de Pinus. A bacia do Bugre foi a que apresentou menor elevação por possuir níveis mais superficiais que tenderam ao alagamento em 2016. Onde a zona não saturada era maior, o armazenamento foi maior e, consequentemente, as elevações nos níveis mais pronunciados. Analisando os outros parâmetros do modelo $(A, a, n, e)$, pode-se observar sistemas de memória curta $(A)$, com rápidas respostas $(a)$, variando em função da distância à drenagem mais próxima e espessura da zona não saturada (n), com forte influência da precipitação, pequena influência da evapotranspiração $(e)$ e com tendências lineares de elevação nos níveis (PTL) entre setembro de 2014 e agosto de 2016.

O modelo PIRFICT tem sido testado e aperfeiçoado ao redor do mundo. Os trabalhos descritos em Von Asmuth (2012) são exemplos de aplicações na Holanda por mais de dez anos de pesquisa e desenvolvimento do modelo. Obergfell et al. (2013) avança nas aplicações do PIRFICT na Holanda utilizando dados de campo. Manzione et al. (2010) testou com sucesso o modelo PIRFICT em área de Cerrado sob diferentes domínios e sistemas hidrogeológicos na região de Planaltina (DF). Yihdego e Webb (2011) apresentam estudo de series temporais usando o modelo PIRFICT na região subúmida do sudoeste da Austrália, ressaltando o bom desempenho do modelo.

De posse do PTL calculado para cada poço, o cálculo do variograma foi realizado utilizando como distância inicial o valor de metade do campo amostral considerado (8,0 Km na direção leste-oeste). Dividiu-se 4,0 Km em 10 passos (lags) de 400,00 metros. A partir desse valor inicial, foram testadas novas configurações de distância do campo amostral e tamanho dos passos. A Tabela 2 apresenta os parâmetros do variograma ajustados para PTL. A Figura 4 apresenta o variograma do ajuste realizado. Para PTL 
foi ajustado um modelo exponencial. Do valor total do patamar encontrado, o efeito pepita foi responsável por 7,69\% do total da variância. O alcance para tendências de elevação foi de 1.765,00 m.

Tabela 2 - Parâmetros do variograma ajustado para os parâmetros de tendência linear (PTL) calculados a partir do modelo PIRFICT

\begin{tabular}{cccccccc}
\hline Variável & $\begin{array}{c}\text { Número de } \\
\text { amostras }\end{array}$ & $\begin{array}{c}\text { Número de } \\
\text { passo }\end{array}$ & $\begin{array}{c}\text { Tamanhos } \\
\text { dos passos } \\
(\mathbf{m})\end{array}$ & $\begin{array}{c}\text { Efeito } \\
\text { Pepita }\end{array}$ & Patamar & Alcance (m) & Modelo \\
\hline$P T L$ & 32 & 12 & 321,00 & 0,03 & 0,39 & $1.765,00$ & Exponencial \\
\hline \multicolumn{7}{c}{ Fonte: Organizado pelo autor. }
\end{tabular}

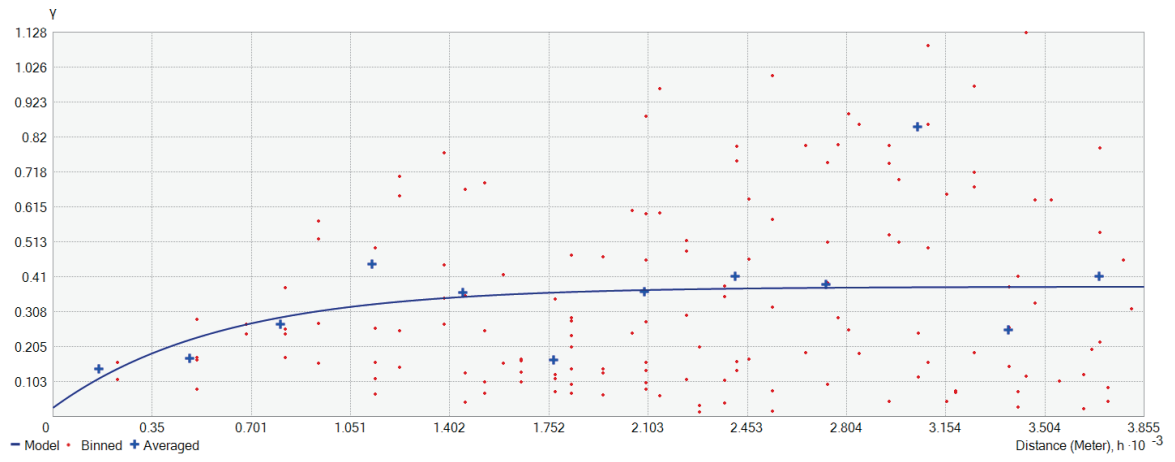

Figura 4 - Variograma amostral e modelo teórico ajustado aos valores de tendências de elevação (Parâmetro de Tendência Linear) calculados pelo modelo PIRFICT

Fonte: Organizado pelo autor.

O mapeamento de tendências de elevação entre setembro de 2014 e agosto de 2016 pode ser visto na Figura 5. As tendências de elevação interpoladas variaram de $28 \mathrm{~cm}$ a 2,17 metros. As maiores elevações se deram nas bacias do Guarantã e Santana, que confluem para o Rio Capivari e forma uma série de áreas úmidas e alagadas, restringindo o fluxo local das águas superficiais e, consequentemente, alterando a dinâmica das águas subterrâneas. Nas Bacias do Bugre e Passarinho as elevações foram menores, demonstrando a capacidade das bacias em drenar as águas subterrâneas e produzir água para alimentar o Rio Capão Rico. 


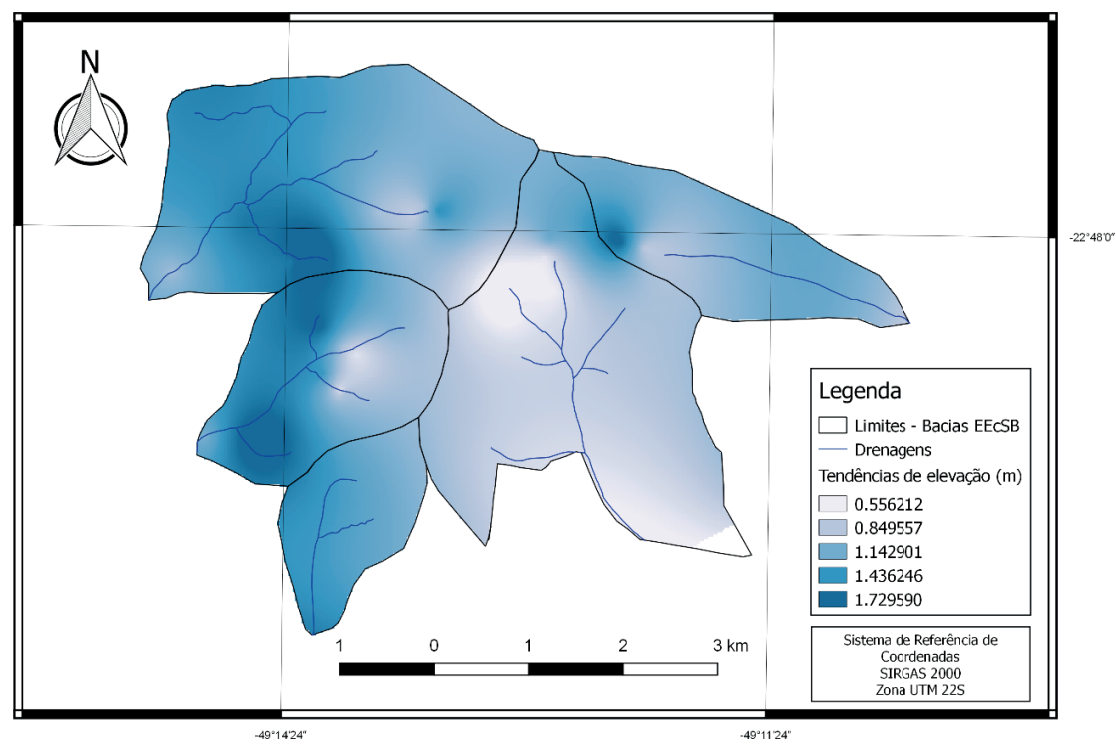

Figura 5 - Mapa de tendência de elevação dos níveis freáticos no período de setembro de 2014 a agosto de 2016 interpolado por krigagem ordinária

Fonte: Organizado pelo autor.

No procedimento de validação cruzada dos resultados, retirou-se o valor amostrado em determinado ponto e obteve-se a estimativa do mesmo por krigagem ordinária, usando os valores dos pontos vizinhos. Considerou-se como melhor estimativa aquela que apresenta média padronizada ( $M S$ - Mean Standardized) próxima de zero, o menor valor possível da raiz da média quadrática (RMS - Root-Mean-Square), a média do desvio padrão ( $A S E$ - Average Standard Error) próxima da média padronizada e o valor da raiz da média quadrática padronizada (RMSS Root-Mean-Square Standardized) próximo de 1. O RMSS é uma medida da acurácia dos valores interpolados. Segundo Johnston et al. (2001), valores de RMSS abaixo de 1 indicam subestimação enquanto valores acima de 1 indicam superestimação dos valores interpolados. Os valores da $M S$ foram próximos a zero e de $A S E$ foram próximas aos valores da RMS. Os valores da RMSS foram todos abaixo de 1, denotando a subestimação dos dados. Isso se deve ao efeito de suavização do interpolador por krigagem 
ordinária, que honra o valor da média, mas acaba apresentando erros nos valores mais extremos da distribuição normal dos dados (Yamamoto; Landim, 2013).

\section{Conclusão}

As principais conclusões reunidas a partir da modelagem dos dados do monitoramento agrohidrometeorológico realizado foram:

- o modelo de FTR em tempo contínuo PRIFICT apresentou bons ajustes, identificando sistemas com uma memória curta, rápida resposta e com forte influência sazonal, principalmente da precipitação;

- a modelagem temporal dos dados de monitoramento revelou padrões de elevação dos níveis no período de setembro de 2014 e agosto de 2016, com a recarga do SAB na área de estudo após a seca 2013/2014, influenciado pelo fenômeno ENOS;

- a análise espacial utilizando técnicas geoestatísticas revelou um padrão espacial das elevações de nível freático no período estudado; e

- fenômeno de oscilação dos níveis freáticos apresentou-se gradual na área da EEcSB, variando suavemente no espaço, sem mudanças abruptas.

\section{Referências}

ALLEN, R. G. et al. Crop evapotranspiration: guidelines for computing crop water requirements. Rome: FAO, 1998. 300 p. (FAO. Irrigation and Drainage Paper, 56). Disponível em: <http://www.fao.org/docrep/X0490E/X0490E00. htm>. Acesso em: 16 mar. 2016.

BECCHINI, L. et al. Modelling, Interpolation and Stochastic simulation in space and time of global solar radiation. Agriculture, ecosystems and Environment, v. 81, p. 29-42, 2000.

BOULANGER, J. P; LELOUP, J.; PENALBA, O.; RUSTICUCCI, M.; LAFON, F.; VARGAS, W. Observed precipitation in the Paraná-Plata hydrological basin: long-term trends, extreme conditions and ENSO teleconnections. Climate Dynamics, v. 24, p. 393-413, 2005.

CBH-MP. Comitê de Bacia Hidrográfica do Médio Paranapanema. As águas do aquífero Bauru no Médio Paranapanema. Marília, SP: Comitê de Bacia Hidrográfica do Médio Paranapanema, 2010. 20 p. 
CEPAGRI. Centro de Pesquisas Meteorológicas e Climáticas Aplicadas a Agricultura. Clima dos Municípios Paulistas. Disponível em: < http://www.cpa. unicamp.br/outras-informacoes/clima-dos-municipios-paulistas.html>. Acesso em: 16 mar. 2016.

COELHO, C. A. S.; CARDOSO, D. H. F.; FIRPO, M. A. F. Precipitation diagnostics of an exceptionally dry event in São Paulo, Brazil. Theoretical and Applied Climatology, v. 125, p. 769-784, 2016.

CPRM. Serviço Geológico do Brasil. Domínio Geológico Litoestratográfico do Estado de São Paulo. São Paulo, 2006. Escala 1:750.000.

CRESSIE, N.; HOLAN, S. H. Editorial: Special issue on time series in the environmental sciences. Journal of Time Series Analysis, v. 32, p. 337-338, 2011.

CRESSIE, N.; WIKLE, C. K. Statistics for spatio-temporal data. Hoboken: John Wiley \& Sons, 2011. 588p.

JOHNSTON, K. et al. Using ArcGIS geostatistical analyst. Redlands: Esri, 2001. $300 \mathrm{p}$.

JOURNEL, A. G.; HUIJBREGTS, C. J. Mining geostatistics. London: Academic Press, 1978. 600 p.

KNOTTERS, M. Regionalised time series models for water table depths. 2001. $97 \mathrm{f}$. Tese (Doutorado em Hidrologia) - Universidade de Wageningen (WUR), Wageningen, 2001. Disponível em: <http://library.wur.nl/WebQuery/wurpubs/ fulltext/121258>. Acesso em: 06 maio 2017.

MANZIONE, R. L. Regionalized spatio-temporal modeling of water table depths in the Brazilian Cerrado. 2007. 141 f. Tese (Doutorado em Sensoriamento Remoto) - Instituto Nacional de Pesquisas Espaciais (INPE), São José dos Campos, 2007. Disponível em: <http://www.dpi.inpe.br/gilberto/teses/tese_rodrigo.pdf $>$. Acesso em: 06 maio 2017.

MANZIONE, R. L. et al. Transfer function-noise modeling and spatial interpolation to evaluate the risk of extreme (shallow) water-table levels in the Brazilian Cerrados. Hydrogeology Journal, v. 18, p. 1927-1937, 2010.

MELO, A. C. G.; DURIGAN, G. Plano de manejo da Estação Ecológica de Santa Bárbara. São Paulo: Instituto Florestal, 2011. 222 p.

MESTRINHO, S. S. P. Monitoramento em água subterrânea. In: FEITOSA, F. A. C.; MANOEL FILHO, J.; FEITOSA, E. C.; DEMETRIO, J. G. A. Hidrogeologia: Conceitos e aplicações. Rio de Janeiro: CPRM/LABHID, 2008. p. 673-686.

MOORE, J. E. Field Hydrogeology. A guide for site investigations and report preparation. London/New York/Boca Raton: CRC Press, 2012. 190p.

OBERGFELL, C. et al. Deriving hydrogeological parameters through time series analysis of groundwater head fluctuations around well fields. Hydrogeology Journal, v. 21, p. 987-999, 2013.

OLIVEIRA, J. B. et al. Mapa Pedológico do Estado de São Paulo. Campinas, SP: IAC/FAPESP, 1999. 1:500.000. 
PENALBA, O. C. et al. ENSO impact on dry sequences during different phenological periods in the east-northeast of Argentina. In: III INTERNATIONAL CONFERENCE ON ENSO: Bridging the gaps between global ENSO science and regional processes, extremes and impacts, Guayaquil, Ecuador, 12-14 November, 2014.

PENALBA, O. C.; RIVERA, J. A. Precipitation response to El Niño/La Niña events in Southern South America - emphasis in regional drought occurrences. Advances in Geosciences, v. 42, p. 1-14, 2016.

PODESTÁ, G. P. et al. Associations between grain crop yields in central-eastern Argentina and El Niño-Southern Oscillation. Journal of Applied Meteorology, v. 38, p. 1488-1498, 1999.

ROSS, J. L. S.; MOROZ, I. C. Mapa geomorfológico do Estado de São Paulo. Revista do Departamento de Geografia, v. 10, p. 41-56, 1996.

SHAPOORI, V. et al. Decomposing groundwater head variations into meteorological and pumping components: a synthetic study. Hydrogeology Journal, v. 23, p. 1431-1448, 2015.

SPESCHA, L. et al. Los excesos de agua edáfica y su relación con el ENSO en la región Pampeana. Rev. Facultad de Agronomía, v. 24, p. 161-167, 2004.

STEIN, A.; STARITISKY, G.; BOUMA, J. simulation of moisture deficits and areal interpolation by universal cokriging. Water Resources Research, v. 27, p. 1963-1973, 1991.

VON ASMUTH, J. R. Groundwater system identification through time series analysis. 2012. 221 f. Tese (Doutorado em Hidrologia) - TU Delft, Delft, 2012.

VON ASMUTH, J. R.; BIERKENS, M. F. P. Modelling irregularly spaced residual series as a continuous stochastic process. Water Resources Research, v. 41, p. W12404, 2005.

VON ASMUTH, J. R.; BIERKENS, M. F. P.; MAAS, C. Transfer function noise modelling in continuous time using predefined impulse response functions. Water Resources Research, v. 38, p. 23.1-23,12. 2002.

VON ASMUTH, J. R. et al. Software for hydrogeologic time series analysis, interfacing data with physical insight. Environmental Modelling and Software, v. 38, p. 178-190, 2012.

VON ASMUTH, J. R.; MAAS, C. The method of impulse response moments: a new method integrating time series, groundwater and eco-hydrological modelling. In: GEHERLS, J. C.; PETERS, N. E.; HOEHN, E.; JENSEN, K.; LEIBUNDGUT, C.; GRIFFIOEN, J.; WEBB, B.; ZAADNOORDIJK, W. J. (Ed.) Impact of human activity on groundwater dynamics. Wallingford: IAHS Publication, 2001. p. 51-58.

YAMAMOTO, J. K.; LANDIM, P. M. B. Geoestatística: conceitos e aplicações. São Paulo: Oficina de Textos, 2013. 215 p.

YIHDEGO, Y.; WEBB, J. A. Modeling of bore hydrographs to determine the impact of climate and land-use change in a temperate subhumid region of southeastern Australia. Hydrogelogy Journal, v. 19, p. 877-887, 2011. 
Artigo

ZIEMER, R. E.; TRANTER, W. H.; FANNIN, D. R. Signals and systems: continuous and discrete. Upper Saddle River: Prentice-Hall, 1998. 622 p.

Rodrigo Lilla Manzione - Possui graduação em Agronomia pela Universidade Estadual Paulista Júlio de Mesquita Filho, mestrado em Agronomia (Energia na Agricultura) pela mesma Universidade e doutorado em sensoriamento remoto pelo Instituto Nacional de Pesquisas Espaciais em São José dos Campos, com período sanduíche no Instituto Alterra (Wageningen, Holanda). Atualmente é professor na Universidade Estadual Paulista Júlio de Mesquita Filho, em regime de dedicação integral à docência e pesquisa. ORCID: http://orcid.org/0000-0002-075 4-2641

Recebido para publicação em 20 de setembro de 2017 Aceito para publicação em 3 de novembro de 2017 